\title{
Spin-dependent electronic structure of transition-metal atomic chains adsorbed on single-wall carbon nanotubes
}

\author{
E. Durgun and S. Ciraci* \\ Department of Physics, Bilkent University, Ankara 06800, Turkey
}

(Received 19 January 2006; revised manuscript received 5 June 2006; published 5 September 2006)

\begin{abstract}
We present a systematic study of the electronic and magnetic properties of transition-metal (TM) atomic chains adsorbed on the zigzag single-wall carbon nanotubes (SWNTs). We considered the adsorption on the external and internal wall of SWNT and examined the effect of the TM coverage and geometry on the binding energy and the spin polarization at the Fermi level. All those adsorbed chains studied have ferromagnetic ground state, but only their specific types and geometries demonstrated high spin polarization near the Fermi level. Their magnetic moment and binding energy in the ground state display interesting variation with the number of $d$ electrons of the TM atom. We also show that specific chains of transition-metal atoms adsorbed on a SWNT can lead to semiconducting properties for the minority spin bands, but semimetallic for the majority spin bands. Spin polarization is maintained even when the underlying SWNT is subjected to high radial strain. Spin-dependent electronic structure becomes discretized when TM atoms are adsorbed on finite segments of SWNTs. Once coupled with nonmagnetic metal electrodes, these magnetic needles or nanomagnets can perform as spin-dependent resonant tunneling devices. The electronic and magnetic properties of these nanomagnets can be engineered depending on the type and decoration of adsorbed TM atom as well as the size and symmetry of the tube. Our study is performed by using first-principles pseudopotential plane wave method within spin-polarized density functional method.
\end{abstract}

DOI: 10.1103/PhysRevB.74.125404

PACS number(s): 73.22.-f, 68.43.Bc

\section{INTRODUCTION}

Nanotubes interacting with magnetic foreign objects have been a focus of attention due to the possibility of realizing the technologically promising area of spintronics in molecular structures. The ability to produce sizeable changes in the conductance of a nanotube due to an applied magnetic field has been one of the driving forces for active research on magnetic properties of carbon-based structures. ${ }^{1,2}$ Due to their inherent spin asymmetry, the interaction with magnetic foreign objects, such as adsorbed transition metal (TM) atoms, ${ }^{3-5}$ nanoparticles, ${ }^{6}$ and substrates, ${ }^{7}$ is likely to cause a spin-dependent response on the transport properties of the combined structure. ${ }^{3,6,8}$ It is now understood that spin-valve effect appears to have potential applications in the development of faster, smaller, and more efficient nanoscale magnetoelectronic devices.

Costa et al. ${ }^{9}$ investigated the indirect magnetic coupling between two distant magnetic adatoms attached to the wall of a carbon nanotube. They found that the coupling between TM atoms is mediated by the electronic carriers and is oscillatory for metallic armchair tubes, but monotonic for zigzag nanotubes. Spin-dependent transport through carbon nanotubes sandwiched between ferromagnetic electrodes has been studied recently. Experimental papers dealing with multiwall carbon nanotubes (MWNT) have produced results which differ not only quantitatively, but also qualitatively from one another. For example, reported maximum GMR values using Co contacts have ranged from $9 \%{ }^{1}$ to $30 \% .^{10}$

The interaction of magnetic atoms with nanotubes may result in a half-metallic (HM) system (namely a metal for one spin direction, but semiconductor for the opposite spin direction) that is of interest for spintronic devices ${ }^{1}$ as well as nanomagnets. Since some carbon nanotubes are ballistic conductors, ${ }^{11,12}$ the spin polarization induced by magnetic electrodes (such as $\mathrm{Fe}, \mathrm{Co}$, or $\mathrm{Ni}$ ) can be preserved as the electrons propagate through the nanotube. To this end, it has been necessary to know which elements can be best bonded to nanotubes and how they affect the magnetic properties. Based on the first-principles density functional theory (DFT) calculation, Yang et al. ${ }^{13}$ found that a $\mathrm{Cr}$ or $\mathrm{V}$ atomic chain adsorbed on a metallic armchair carbon nanotube opens up a band gap for the minority spin states, making the whole system a $100 \%$ spin-polarized conductor. The band gaps of minority spin bands were 0.49 and $0.44 \mathrm{eV}$ for $\mathrm{V}$ and $\mathrm{Cr}$, respectively. The adsorption of $\mathrm{Mn}, \mathrm{Fe}, \mathrm{Co}$, or Ni chains led to large but not complete spin polarization. ${ }^{13}$ Fagan et al. ${ }^{4,5}$ studied the structural, electronic, and magnetic properties of Fe chains adsorbed on SWNT. They discussed several configurations including external and internal geometries by presenting calculated binding energies, band structures, and magnetic moments. Similarly, Yagi et al. ${ }^{14}$ investigated the interaction of $3 d$ transition metal atoms and dimers with a single-walled armchair carbon nanotube by first-principles DFT. They found that Co atoms adsorbed at the hollow site of internal wall of armchair nanotubes can show halfmetallic behavior.

In this paper we present the spin-dependent properties of TM (Co, Cr, Fe, Mn, and V) atomic chains adsorbed on the external and internal walls of zigzag SWNTs. We examined how the spin polarization varies with radius of SWNT as well as with the type of TM atoms, which are adsorbed according to well-defined patterns (decorations). Moreover the strain analysis in radial and axial directions are performed in order to reveal how robust the magnetic properties are. Present work is complementary to other studies, which mainly focused on the metallic armchair nanotubes and predicted half-metallicity. ${ }^{13-15}$ We note that while the half- 
TABLE I. The distance between TM and nearest $\mathrm{C}$ atom $d_{\mathrm{C}-\mathrm{TM}}$; average binding energy $E_{b}$; average magnetic moments per atom $\mu$; spin polarization at the Fermi level $P\left(E_{F}\right)$ for chain structures of $\mathrm{Co}, \mathrm{Cr}, \mathrm{Fe}, \mathrm{Mn}$, and $\mathrm{V}$ transition metal atoms adsorbed on the $(8,0) \mathrm{SWNT}$ for $\theta=1 / 2,1$, and 2. $P\left(E_{F}\right)<$ corresponds to $D\left(E_{F}, \downarrow\right)>D\left(E_{F}, \uparrow\right)$.

\begin{tabular}{|c|c|c|c|c|c|c|c|c|c|c|c|c|}
\hline & \multicolumn{4}{|c|}{$\theta=1 / 2$} & \multicolumn{4}{|c|}{$\theta=1$} & \multicolumn{4}{|c|}{$\theta=2$} \\
\hline & $d_{\mathrm{C}-\mathrm{TM}}(\AA)$ & $E_{b}(\mathrm{eV})$ & $\mu\left(\mu_{B}\right)$ & $P\left(E_{F}\right)$ & $d_{\mathrm{C}-\mathrm{TM}}$ & $E_{b}(\mathrm{eV})$ & $\mu\left(\mu_{B}\right)$ & $P\left(E_{F}\right)$ & $d_{\mathrm{C}-\mathrm{TM}}$ & $E_{b}(\mathrm{eV})$ & $\mu\left(\mu_{B}\right)$ & $P\left(E_{F}\right)$ \\
\hline Co & 2.0 & 1.7 & 1.1 & - & 2.0 & 1.4 & 1.1 & - & 2.0 & 0.6 & 1.7 & -0.65 \\
\hline $\mathrm{Cr}$ & 2.2 & 0.6 & 4.2 & -0.21 & 2.2 & 0.4 & 5.2 & 0.38 & 2.3 & 0.5 & 4.4 & 0.53 \\
\hline $\mathrm{Fe}$ & 2.1 & 0.8 & 2.2 & -0.91 & 2.1 & 0.9 & 4.0 & - & 2.2 & 0.5 & 3.1 & -0.65 \\
\hline Mn & 2.2 & 0.4 & 5.5 & - & 2.2 & 0.7 & 5.0 & - & 2.5 & 0.6 & 4.6 & -0.19 \\
\hline $\mathrm{V}$ & 2.2 & 1.4 & 3.8 & 0.68 & 2.2 & 1.5 & 4.1 & 0.90 & 2.3 & 1.0 & 2.9 & 0.73 \\
\hline
\end{tabular}

metallicity requiring an integer number of spin per unit cell can exist only for infinite and ideal systems, realistic devices can be produced only on finite-size SWNTs, which are either connected to the metal leads or lie on a substrate. In this respect, the main issue is to achieve a high spin polarization on a finite size SWNT. In order to clarify the effect of nanotube size on the spin-dependent electronic structure, we also examined finite systems.

\section{METHOD}

We have performed first-principles plane wave calculations $^{16,17}$ within DFT $^{18}$ using ultrasoft pseudopotentials. ${ }^{19}$ The exchange-correlation potential has been approximated by generalized gradient approximation (GGA) using two different functionals, PW91 ${ }^{20}$ and PBE. ${ }^{21}$ For partial occupancies, we have used the Methfessel-Paxton smearing method. ${ }^{22}$ The width of smearing has been chosen as $0.1 \mathrm{eV}$ for geometry relaxations and $0.01 \mathrm{eV}$ for accurate energy band and density-of-state (DOS) calculations. All structures have been treated by supercell geometry (with lattice parameters $a_{s c}, b_{s c}$, and $c_{s c}$ ) using the periodic boundary conditions. A large spacing $(\sim 10 \AA)$ between adjacent nanotubes has been assured to prevent interactions between them. ${ }^{23}$ In single-cell calculations of infinite systems, $c_{s c}$ has been taken to be equal to the lattice parameter of SWNT and in double-cell calculations, $c_{s c}=2 c$. Convergence with respect to the number of plane waves used in expanding Bloch functions and $\mathbf{k}$ points used in sampling the Brillouin zone (BZ) have been tested before analyzing the systems. ${ }^{24}$ In the self-consistent potential and total energy calculations the BZ of nanotubes has been sampled by $(1 \times 1 \times 15)$ and $(1 \times 1 \times 11)$ mesh points in $\mathbf{k}$ space within Monkhorst-Pack scheme $^{25}$ for single and double cells, respectively. A planewave basis set with kinetic energy cutoff $\hbar^{2}|\mathbf{k}+\mathbf{G}|^{2} / 2 m$ $=350 \mathrm{eV}$ has been used. All atomic positions and lattice parameters have been optimized by using the conjugate gradient method where total energy and atomic forces are minimized. The convergence of calculation is achieved when the difference of the total energies of the last two consecutive steps is less than $10^{-5} \mathrm{eV}$, and the maximum force allowed on each atom is less than $0.05 \mathrm{eV} / \AA$. As for finite structures, supercell has been constructed in order to yield $\sim 10 \AA$ vacuum space in each direction, and BZ is sampled only at the $\Gamma$ point. The other parameters of the calculations are kept the same. The binding energy (per atom) of the adsorbed TM atomic chain has been calculated for each configuration by using the expression

$$
\begin{aligned}
E_{b}= & \left\{E_{T}[\mathrm{SWNT}]+E_{T}[\mathrm{TM}-\text { chain }]\right. \\
& \left.-E_{T}[\mathrm{SWNT}+\mathrm{TM}-\text { chain }]\right\} / N,
\end{aligned}
$$

where $N$ is the number of adsorbed TM atoms per cell. In this equation, three terms, respectively, stand for the optimized total energy of the bare SWNT, TM-chain, and SWNT with adsorbed TM-chain. All the optimized total energies are calculated in the same supercell. The spin polarization at the Fermi level, $E_{F}$ is defined as

$$
P\left(E_{F}\right)=\left[D\left(E_{F, \uparrow}\right)-D\left(E_{F, \downarrow}\right)\right] /\left[D\left(E_{F, \uparrow}\right)+D\left(E_{F, \downarrow}\right)\right]
$$

in terms of the density of states of majority and minority spin states, $D\left(E_{F, \uparrow}\right)$ and $D\left(E_{F, \downarrow}\right)$, respectively. The average binding energy $E_{b}$, the average magnetic moment per adsorbed TM atom $\mu$, and $P\left(E_{F}\right)$ have been calculated for different levels of coverage of $\theta=1 / 2,1$, and 2. Here $\theta$ indicates the number of adsorbed TM atoms per unit cell. In order to remove the constraints of supercell geometry and to test the stability further, the TM atomic chain SWNT systems have been relaxed after their supercell sizes are doubled (namely $c_{s c}=2 c$ ). Moreover, in order to test the effects of deformation on the physical properties, we have also studied the cases where the underlying $(8,0)$ tubes are kept under $25 \%$ radial strain. ${ }^{26}$

\section{TM WIRES ADSORBED ON SWNT}

In this section, we first summarize our results for TM atoms adsorbed on the external and internal walls of the $(8,0)$ SWNT to form an atomic chain.

\section{A. External adsorption}

Bond distances $d, E_{b}, \mu$, and $P\left(E_{F}\right)$ calculated for the Co, $\mathrm{Cr}, \mathrm{Fe}, \mathrm{Mn}$, and $\mathrm{V}$ atomic chains adsorbed on the $(8,0)$ zigzag SWNT are listed in Table I for $\theta=1 / 2$, 1, and 2. The atomistic model corresponding to various coverages is illustrated in Fig. 1. The spin-polarized band structures and the total density of states (TDOS) near $E_{F}$ are presented in Fig. 2 


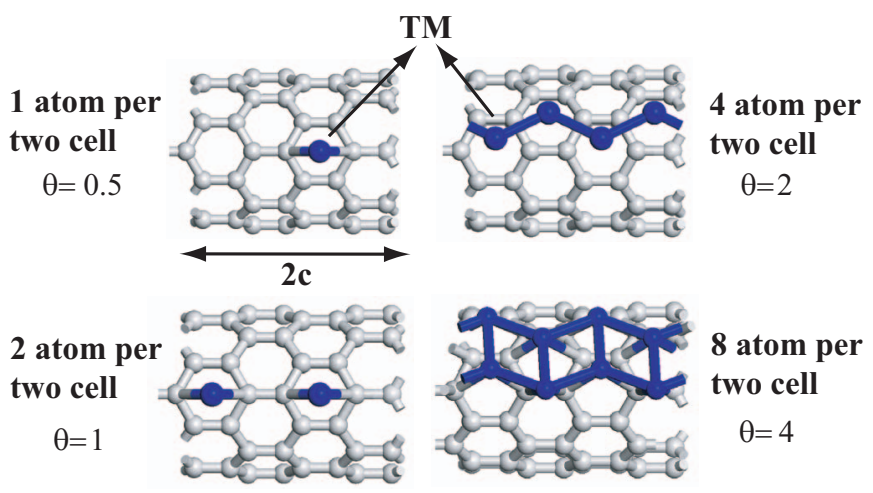

FIG. 1. (Color online) The configuration of adsorbed TM atoms ( Co, Cr, Fe, Mn, and V) forming chain structures on the $(8,0)$ SWNT are illustrated for various coverage geometries, such as $\theta=1 / 2,1,2$, and 4 .

for adsorbed V, Co, and Fe chains and in Fig. 3 for Mn and Cr chains.

\section{Vanadium}

The ground state of the V chain adsorbed on SWNT is found to be ferromagnetic for all geometries described in Fig. 1. The value of the average magnetic moment, ${ }^{27} \mu$ is calculated as 3.8, 4.1, and $2.9 \mu_{B}$ for $\theta=1 / 2,1$, and 2 , respectively. The calculations are also performed for the bare $\mathrm{V}$ chains by removing the $(8,0) \mathrm{SWNT}$, but keeping the same chain geometry when they were adsorbed on the tube. For example, for $\theta=1 / 2$, where the distance between nearest $\mathrm{V}$ atoms $\left(d_{\mathrm{V}-\mathrm{V}}\right)$ is $8.52 \AA, \mu$ is calculated as $5.0 \mu_{B}$ that is equal to the magnetic moment of the free $\mathrm{V}$ atom in $s^{1} d^{4}$ configuration. This indicates that for $\theta=1 / 2 \mathrm{~V}-\mathrm{V}$ coupling is negligible. $\mathrm{V}-\mathrm{C}$ interaction or charge transfer between $\mathrm{V}$ and $\mathrm{C}$ atoms is responsible for the reduction of $\mu$ from
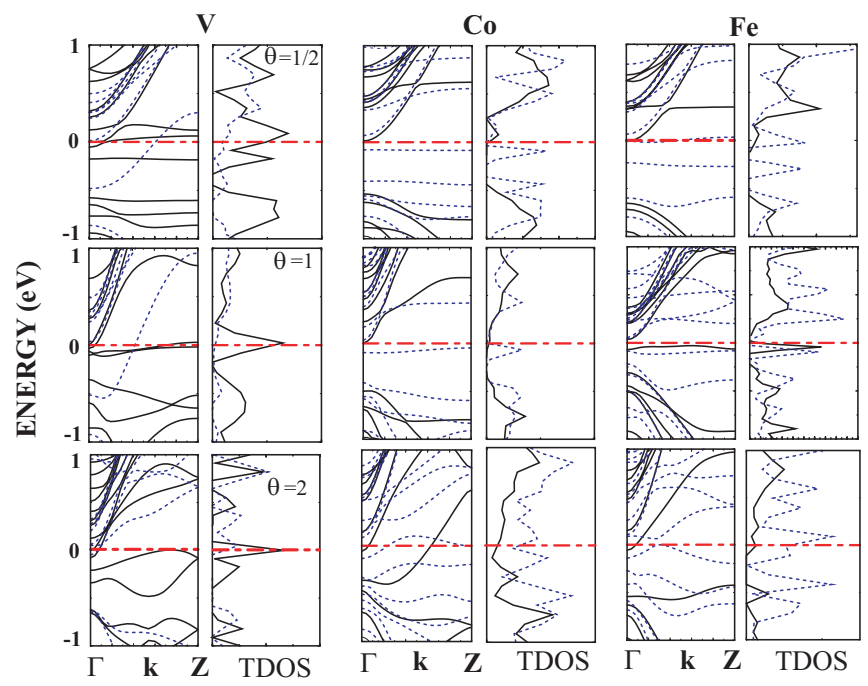

FIG. 2. (Color online) The spin-dependent band structure and TDOS of $\mathrm{V}, \mathrm{Co}$, and $\mathrm{Fe}$ chains adsorbed on the zigzag $(8,0)$ SWNT for $\theta=1 / 2,1$, and 2 geometries. Solid and dotted lines are for majority and minority spin states, respectively. The zero of energy is set to the Fermi level $E_{F}$.
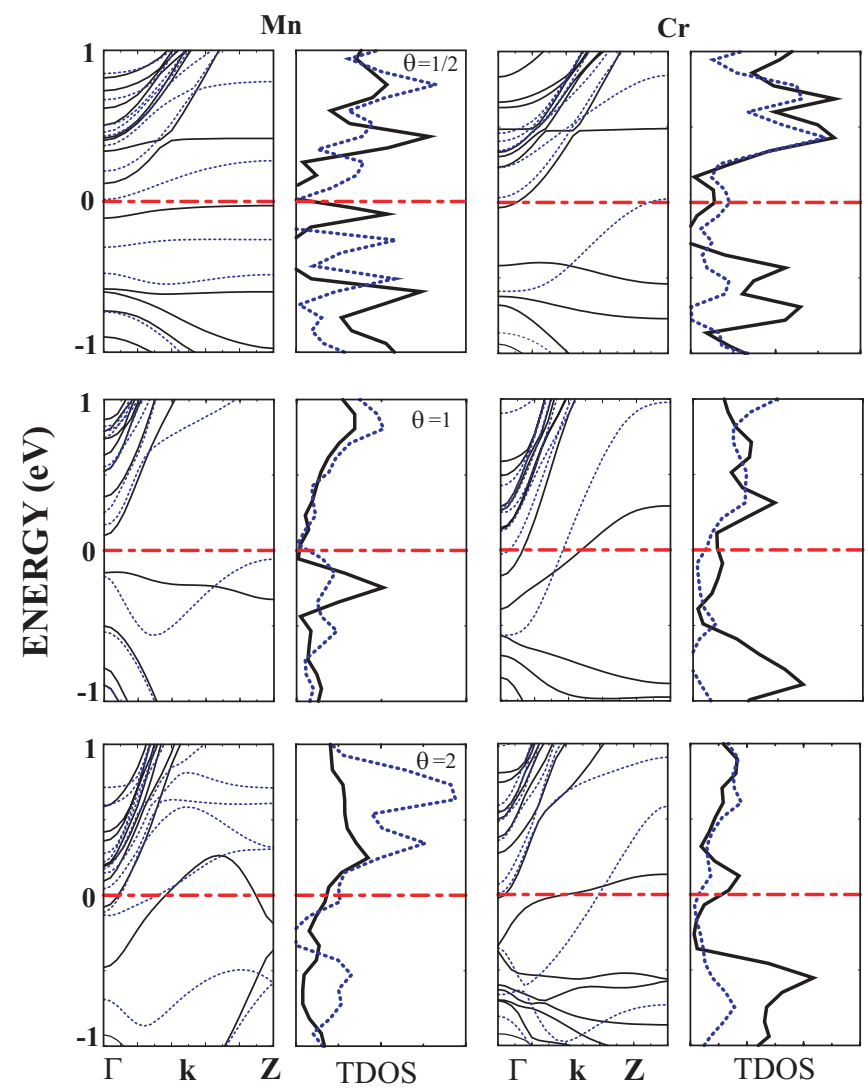

FIG. 3. (Color online) The spin-dependent band structure and TDOS of Mn and $\mathrm{Cr}$ chains adsorbed on the zigzag $(8,0)$ SWNT for $\theta=1 / 2,1$, and 2 geometries. Solid and dotted lines are for majority and minority spin states, respectively. The zero of energy is set to the Fermi level $E_{F}$.

$5.0 \mu_{B}$ to $3.8 \mu_{B}$ upon adsorption on SWNT. As for $\theta=1$, $d_{\mathrm{V}-\mathrm{V}}$ of the bare $\mathrm{V}$ atomic chain becomes $4.26 \AA$ and $\mu$ $=4.8 \mu_{B}$; for $\theta=2, d_{\mathrm{V}-\mathrm{V}}=2.4 \AA$ and resulting $\mu$ is $4.1 \mu_{B}$. These interactions are also crucial for the stability of decorated structures on SWNT which were discussed previously both experimentally ${ }^{28}$ and theoretically. ${ }^{8}$ The coupling between $\mathrm{V}$ atoms gets stronger as $\theta$ increases. This causes a slight increase in the distance between $\mathrm{V}-\mathrm{C}$ atoms from 2.2 to $2.3 \AA$.

The adsorption of the $\mathrm{V}$ chain makes the semiconducting $(8,0)$ SWNT metallic for both spin directions. Complete polarization at $E_{F}$, in other words half-metallicity (having an integer number of net spin in a cell) did not occur. However, for $\theta=1 / 2$ and 1 the density of states for majority spin carrier at $E_{F}, D\left(E_{F}, \uparrow\right)$ is much larger than minority spin carrier as illustrated in Fig. 2. Since, $P\left(E_{F}\right)$ as large as $90 \%$ can be achieved, these structures may be suitable for spintronic device applications.

For nanotubes, namely $(10,0)$ and $(14,0)$ with a larger radius, Yang et al. ${ }^{13}$ found $d_{\mathrm{C}-\mathrm{V}}$ as $2.2 \AA$ and $\mu=2.2 \mu_{B}$ with $P\left(E_{F}\right)=45 \%$ for $\theta=2$ geometry. They also showed that $\mathrm{V}$ chains adsorbed on armchair SWNTs exhibit HM properties. ${ }^{13}$ Andriotis et al. ${ }^{29}$ found that the hollow site of graphene $\mathrm{e}^{30,31}$ is energetically favorable with $d_{\mathrm{C}-\mathrm{V}} \sim 1.9 \AA$ and $\mu=1.02 \mu_{B}$. 


\section{Iron}

For $\theta=1 / 2$, the SWNT+TM atomic chain system has a ferromagnetic ground state with $\mu=2.2 \mu_{B}$. Here $\mu$ is reduced from the magnetic moment of free atom due to $\mathrm{Fe}-\mathrm{C}$ interaction which, in turn, results in transfer of $4 s$ electrons to $3 d$ as confirmed by our Mulliken analysis. The energy band calculation shows that the system is metallic for both types of spin carriers, but $P\left(E_{F}\right)$ is very high for minority spin carriers. Analysis of partial density of states (PDOS) suggests that the hybridized $3 d$ states of Fe contribute to $P\left(E_{F}\right)$. For $\theta=1$, the ground state of the system is still ferromagnetic, but the increased $\mathrm{Fe}-\mathrm{Fe}$ interaction and reduction in unit cell size make the system semiconducting with negligible $P\left(E_{F}\right)$. For $\theta=2$, the ferromagnetic system is metallic for both spin carriers. While $\mathrm{Fe}-\mathrm{C}$ distances have changed slightly under radial strain $\epsilon_{r}=-0.25$, the metallicity for both spin carriers and high $P\left(E_{F}\right)$ is maintained. For the same structure on the $(8,0)$ SWNT, Fagan et al. ${ }^{4}$ obtained similar results for ground state properties. They calculated $d_{\mathrm{C}-\mathrm{Fe}}$ between $2.1-2.4 \AA$ and $\mu$ as $3.0 \mu_{B}$ which are consistent with present results. However, they obtained $E_{b}$ as $0.9 \mathrm{eV}$ which is larger than ours. Our results indicate that the system is metallic with high $P\left(E_{F}\right)$, whereas they predicted a semiconducting structure with a small gap. Moreover, the optimized geometry of the present study is also different. Those differences between the present study and that of Fagan et al. $^{4}$ perhaps originate from different method of calculations (plane wave vs local basis set). Yang et $a l .{ }^{13}$ predicting metallic character with $P\left(E_{F}\right)=86 \%$ and $\mu=2.6 \mu_{B}$ for $(14,0)$ confirms our results. For the graphene structure Yagi et al. ${ }^{13}$ and Duffy et al. ${ }^{32}$ also found hollow site as the most stable adsorption site for a single Fe with $\mu \sim 2 \mu_{B}$.

Finally, we have studied the properties of two parallel Fe chains adsorbed on the $(8,0)$ SWNT which is specified as $\theta=4$. The ground state of the system is ferromagnetic with $\mu=3.0 \mu_{B}$ per Fe atom and shows metallic behavior for both spin carriers with negligible $P\left(E_{F}\right)$. Since Fe-Fe interaction is stronger than $\mathrm{Fe}-\mathrm{C}$ interaction the $\mathrm{Fe}$ atoms show a tendency to form a cluster. However, for a different geometry, but the same $\theta$, where two parallel Fe chains are separated (hence Fe-Fe coupling is reduced) the net magnetic moment of the ground state did not change significantly, but $P\left(E_{F}\right)$ increased to $P\left(E_{F}\right)=0.82$. This result suggests that the spin polarization is strongly dependent on $\theta$ as well as on the pattern of the decoration of the adsorbed TM atoms.

The calculations have been repeated by using different GGA functional, namely $\mathrm{PBE}^{21}$ for the Fe-atomic chains adsorbed externally with $\theta=1 / 2,1$, and 2 . The use of PBE did not change the results obtained by using PW91. ${ }^{33}$ For example maximum changes in the binding energy have been $0.1,0.1$, and $0.2 \mathrm{eV}$ for $\theta=1 / 2,1$, and 2 , respectively.

\section{Cromium}

$\mathrm{Cr}$ atomic chains adsorbed on the $(8,0)$ SWNT with $\theta=1 / 2$ and 1 give rise to metallic state for both types of spin carriers and result in negligible $P\left(E_{F}\right)$. However the induced $\mu$ is large. Bare chains of both $\theta=1 / 2$ and 1 have the same $\mu=6 \mu_{B}$ as that of free $\mathrm{Cr}$ atoms in $s^{1} d^{5}$ configuration due to negligible $\mathrm{Cr}-\mathrm{Cr}$ interaction. The decrease in $\mu$ when $\mathrm{Cr}$ is adsorbed on SWNT is due to transfer of $s$ electrons into $d$ electrons. For $\theta=2$ we obtained a significant $P\left(E_{F}\right)$ with $\mu=4.4 \mu_{B}$. Yagi et al. ${ }^{14}$ reported $P\left(E_{F}\right)=29 \%$ with $\mu$ $=3.2 \mu_{B}$ for $(14,0)$ suggesting that $\mu$ decreases with increase of nanotubes radius. The calculations by Yagi et al. ${ }^{14}$ on the $(6,6)$ and $(8,8)$ armchair SWNT showed that a small gap opens for minority spin carriers and system becomes HM with $100 \%$ spin polarization with $\mu=4 \mu_{B}$. They obtained $E_{b}$ less than $1 \mathrm{eV}$ for both metallic and zigzag nanotubes which is consistent with our result indicating relatively weak interaction between Cr and SWNT. Duffy et al. ${ }^{32}$ studied single $\mathrm{Cr}$ adsorption on graphene and reported ferromagnetic ground state with $\mu=5.0 \mu_{B}$.

\section{Cobalt}

While the Co chains adsorbed on the $(8,0)$ SWNT have ferromagnetic ground states with $\mu \sim 1.0 \mu_{B}$ for $\theta=1 / 2$ and 1 , the magnetic moments corresponding to the bare Co chains $\left(\mu=3.0 \mu_{B}\right)$ are equal to that of a single, free Co atom in the $d^{8} s^{1}$ configuration. This indicates that direct Co-Co interaction is almost negligible at distances larger than $4.26 \AA$, but Co-C interaction reduces the strength of $\mu$. The energy band analysis indicates that the Co atomic chains adsorbed on the $(8,0)$ SWNT for $\theta=1 / 2$ and 1 is semihalf metallic, namely the system is semiconducting for minority spin bands, but the band of majority spin states just touches $E_{F}$ at the center of BZ. Moreover, the band originating from localized $3 d_{\downarrow}$ state just below $E_{F}$ contributes to the conductance under small bias and makes that spin polarization significant for minority spin carriers. For $\theta=2$, the system is metallic for both spin directions with high $P\left(E_{F}\right)$ in favor of minority spin carrier. The calculations by Yang et al. ${ }^{13}$ indicated also significant spin polarization with $P\left(E_{F}\right)=41 \%$ with $\mu=1.2 \mu_{B}$ for the Co-chain adsorbed on the $(14,0)$ SWNT for $\theta=2$. The decrease of $\mu$ with increasing radius of zigzag nanotubes is consistent with the results obtained for $\mathrm{V}, \mathrm{Fe}$. The calculations concerning the interaction of $\mathrm{Co}$ atom with $(4,4)$ and $(8,8)$ metallic SWNTs at $\theta=1 / 2$ indicate that even complete polarization at $E_{F}$ can be obtained. ${ }^{30}$

\section{Manganese}

The Mn chain adsorbed on the $(8,0)$ SWNTs has ferromagnetic ground state for $\theta=1 / 2,1$, and 2 geometries. The corresponding magnetic moments $\mu$ per atom are 5.5, 5.0, and $4.6 \mu_{B}$ for $\theta=1 / 2,1$, and 2 , respectively. For $\theta=1 / 2$ geometry, the magnetic moment of the SWNT+Mn chain system is even larger than that of free Mn atom in $s^{1} d^{6}$ configuration. Our PDOS analysis suggests that $\mathrm{Mn}-\mathrm{C}$ interaction through the electron transfer from Mn $4 s$ to $\mathrm{Mn} 3 d$ and $4 p$ is enhancing the spin alignment. ${ }^{5}$ Even the calculations on the interaction of a single $\mathrm{Mn}$ atom with graphene result in a similar charge transfer from $\mathrm{Mn} 4 s$ to $\mathrm{Mn} 4 p$ and $3 d$ orbitals. ${ }^{32}$ Bare Mn chains corresponding to both $\theta=1 / 2$ and $\theta=1$ have magnetic moments equivalent to that of free $\mathrm{Mn}$ atom, since $\mathrm{Mn}-\mathrm{Mn}$ interaction is almost negligible for $d_{\mathrm{Mn}-\mathrm{Mn}}>4 \AA$.

The band gap of the bare $(8,0)$ SWNT increases upon the adsorption of the $\mathrm{Mn}$ chain of $\theta=1 / 2$. The interesting point 

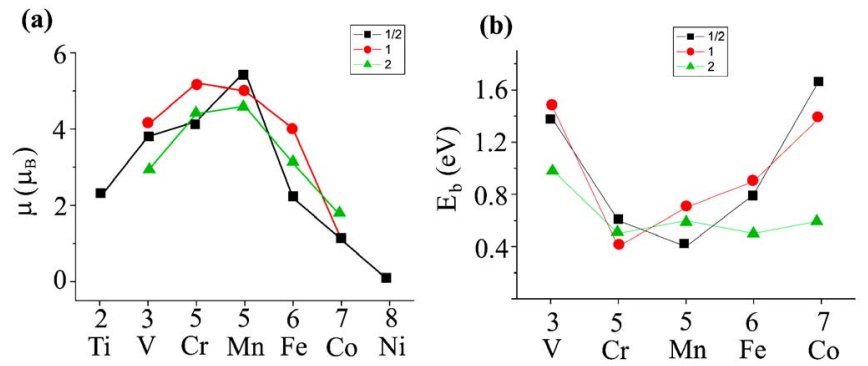

FIG. 4. (Color online) The variation of (a) magnetic moment $\mu$, and (b) the binding energy $E_{b}$ as a function of number of $d$ states for $\theta=1 / 2,1$, and 2 for external adsorption.

is that a band of majority spin states just touches the $E_{F}$ at the $\Gamma$ point exhibiting a half semimetallic character. However, the system is semiconducting for $\theta=1$, but metallic for $\theta=2$ with small $P\left(E_{F}\right)$. For $\theta=2$, Fagan et al. ${ }^{5}$ predicted similar optimized configuration with $\mu=4.2 \mu_{B}$. Yang et al. ${ }^{13}$ reported very high polarization, $P\left(E_{F}\right)=78 \%$ with $\mu=3.6 \mu$ for the case of an Mn chain adsorbed on the $(14,0)$ SWNT according to $\theta=2$.

We finally summarize the general trends revealed from the above discussion. (i) The bond length $d_{\mathrm{C}-\mathrm{TM}}$ ranges between $2.0 \AA$ and $2.5 \AA$; it does not exhibit significant variation with nanotube radius $(\mathrm{R})$. However, $d_{\mathrm{C}-\mathrm{TM}}$ slightly increases with increasing $\theta$ due to the increasing adatomadatom coupling. (ii) The binding energy $E_{b}$ decreases as $R$ increases. This is an expected result due to the curvature effect. $E_{b}$ has the lowest value for Mn having half-filled $d$ shell in $3 d^{5} 4 s^{2}$ configuration. (iii) Generally $\mu$ decreases as $R$ increases. Maximum of $\mu$ is obtained for $\mathrm{Cr}$ and $\mathrm{Mn}$. The variation of $\mu$ and $E_{b}$ with respect to the number of $d$ electrons, $N_{d}$, of the adsorbed TM atom is plotted in Fig. 4. Interestingly, different adsorption geometries corresponding to $\theta=1 / 2,1$, and 2 display similar overall behaviors. The ground state magnetic moment $\mu$ has a maximum value for $N_{d}=5$ (corresponding to half-filling of the $d$ shell). In contrast, $E_{b}$ shows a minimum at $N_{d}=5$. Earlier it has been shown that $E_{b}\left(N_{d}\right)$ passes through a two maxima for $N_{d}=2$ (Ti $3 d^{2} 4 s^{2}$ configuration) and $N_{d}=8$ (Ni in $3 d^{8} 4 s^{2}$ configuration). ${ }^{8}$ The behavior illustrated in Fig. 4 is explained by using Friedel model (see Refs. 8 and 34).

\section{B. Internal adsorption}

The results obtained from the adsorption of $\mathrm{Co}, \mathrm{Fe}$, and $\mathrm{V}$ chain for $\theta=1 / 2$ and 1 on the internal wall of the $(8,0)$
SWNT are summarized in Table II. The variation of $d_{\mathrm{C}-\mathrm{TM}}$, $E_{b}$, and $\mu$ with $\theta$ exhibit trends similar to those in the case of external adsorption. However, spin-polarization at $E_{F}$ displays some differences from external adsorption. For example, while $P\left(E_{F}\right)$ is usually significant for external adsorption at $\theta=1 / 2$, it is negligible for the internal case. Similar to the external counterpart, the ground state of internally doped $(8,0)$ SWNT is ferromagnetic for all geometries. However, the band structure corresponding to the internal adsorption usually changes significantly for most of the cases. This situation shows the effect of confinement on the interaction between TM and C. Both $\theta=1 / 2$ and $\theta=1$ geometries of adsorbed $\mathrm{V}$ chains exhibit metallic character, but the high $P\left(E_{F}\right)$ calculated for the external doping case diminishes for $\theta=1 / 2$ and reduces to 0.22 for $\theta=1$.

The change in $\mu$ is more significant when Fe is adsorbed internally. For $\theta=1 / 2$, while the Fe chain externally doped is metallic with high $P\left(E_{F}\right)$, internally adsorbed system becomes semiconducting. On the other hand, for $\theta=1$, the semiconducting system of the external adsorption case shows metallic behavior with $P\left(E_{F}\right)=-0.62$ for the internal adsorption. The change in electronic structure as well as in $P\left(E_{F}\right)$ is again due to the hybridization of $d$ bands. Localized and almost dispersionless $d$ bands of external chains are dispersed for the internal case due to increased coupling. The overall shape of the band structures is similar, but near $E_{F}$ changes become significant. For the $(4,4)$ armchair SWNT, Yagi et al. ${ }^{14}$ also found ferromagnetic ground state with the same adsorption geometry corresponding to $\theta=1 / 2$. The atomic positions and $E_{b}$ are very close for both cases, but just a small increase in $\mu$ (from 3.0 to $3.1 \mu_{B}$ ) for the internal adsorption is pointed out.

As for $\mathrm{Co}$, the semihalf metallic system becomes semiconducting for $\theta=1 / 2$ and metallic for $\theta=1$ with $P\left(E_{F}\right)=0.77$ in the case of internal adsorption. The change in the dispersion of the $d$ band of minority carriers determines the electronic properties and polarization of the system. The internal adsorption of Co atoms inside $(4,4)$ and $(8,8)$ armchair SWNT makes the system half-metallic. ${ }^{14}$

Briefly, in the internal adsorption, we see that geometry and $d_{\mathrm{C}-\mathrm{TM}}$ do not change significantly with the type of TM atom. $\mu$ generally decreases for the internal adsorption (except for Co), since more $4 s$ electrons are transferred to $3 d$. As the strength of interaction changes, the value of $E_{b}$ oscillates and hinders the derivation of a general rule. Nevertheless, the cases studied here clearly indicate that the polarization near $E_{F}$ can also be manipulated by changing the doping from external to internal walls of SWNT.

TABLE II. The distance between TM and nearest neighbor $\mathrm{C}$ atom $d_{\mathrm{C}-\mathrm{TM}}$, binding energy $E_{b}$, magnetic moments $\mu$ per TM atom, and polarization at Fermi level $P\left(E_{F}\right)$ of various chain structures of $\mathrm{Co}, \mathrm{Fe}$, and $\mathrm{V}$ atoms adsorbed inside the $(8,0)$ SWNT for $\theta=1 / 2$ and $\theta=1$. $P\left(E_{F}\right)<$ corresponds to $D\left(E_{F}, \downarrow\right)>D\left(E_{F}, \uparrow\right)$.

\begin{tabular}{ccccccccc}
\hline \hline & \multicolumn{3}{c}{$\theta=1 / 2$} & \multicolumn{5}{c}{$\theta=1$} \\
\cline { 2 - 8 } & $d_{\mathrm{C}-\mathrm{TM}}(\AA)$ & $E_{b}(\mathrm{eV})$ & $\mu\left(\mu_{B}\right)$ & $P\left(E_{F}\right)$ & $d_{\mathrm{C}-\mathrm{TM}}$ & $E_{b}(\mathrm{eV})$ & $\mu\left(\mu_{B}\right)$ & $P\left(E_{F}\right)$ \\
\hline $\mathrm{Co}$ & 2.0 & 1.2 & 1.0 & - & 2.0 & 1.6 & 1.4 & 0.77 \\
$\mathrm{Fe}$ & 2.2 & 0.4 & 2.3 & - & 2.1 & 0.4 & 2.3 & -0.62 \\
$\mathrm{~V}$ & 2.2 & 1.5 & 3.6 & - & 2.2 & 1.4 & 3.8 & 0.22 \\
\hline \hline
\end{tabular}



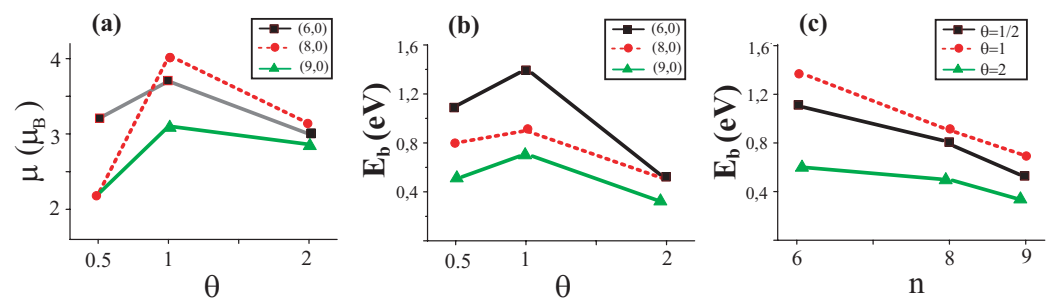

FIG. 5. (Color online) Variation of the average magnetic moment $\mu$ and binding energy $E_{b}$ of an Fe chain adsorbed on the external walls of zigzag $(n, 0)$ SWNT $(n=6,8$, and 9) with the coverage geometry $\theta$ and tube index $n$. (a) $\mu$ versus $\theta$; (b) $E_{b}$ versus $\theta$; (c) $E_{b}$ versus $n$.

\section{Other type of SWNTs}

In addition to the zigzag $(8,0)$ SWNT, we have analyzed the interaction of Fe with the $(6,0)$ and $(9,0)$ tubes which are chosen as prototype for $(n, 0)$ (where $n$ is the integer multiples of 3). The $(6,0) \mathrm{SWNT}$ is metallic due to the dipping of the $\pi^{*}$-singlet conduction band into the valence band as a result of curvature effect. ${ }^{30}$ The $(9,0)$ tube is semiconductor with very small band gap. ${ }^{35}$ All Fe chains $(\theta=1 / 2,1$, and 2$)$ have magnetic ground state. Variation of $\mu$ with $\theta$ is illustrated in Fig. 5(a). $\theta$ as well as with the index of SWNT $(n)$ are shown in Figs. 5(b) and 5(c). It appears that $\mu(\theta)$ exhibits similar variation with $\theta$ for $n=6,8$, and 9. $\mu(\theta=2)$ has comparable values for all three tubes which have different radii. Owing to the curvature effect, $E_{b}$ increases as $n$ decreases (or $R$ decreases). Their electronic band structures also display interesting properties. For $(6,0)$, the system is metallic for all $\theta$ and shows high $P\left(E_{F}\right)$ except $\theta=1$. For $(9,0)$, the minority spin bands just touch $E_{F}$ and a small gap opens for majority spin bands and the system becomes almost half-metallic with perfect spin polarization at $\theta=1 / 2$ and 2 . For $\theta=1, P\left(E_{F}\right)$ is also high in favor of minority spin carriers but the system becomes metallic for minority and majority spin bands.

\section{Adsorption on finite tubes}

While the study of periodic or infinite structures in previous sections may give an idea about the behavior of the systems in ideal cases, devices in real applications should have finite size and may be on substrates and/or connected to the leads. To examine the finite size effect, we considered an Fe chain adsorbed on the finite $(8,0)$ tube. In the first model, we placed $\mathrm{Fe}$ atoms according to $\theta=1 / 2$ and 1 geometry on a segment of the $(8,0)$ SWNT consisting of 64 carbon atoms and for the $\theta=2$ case on a segment consisting of 128 carbon atoms. All tubes have open but fixed ends. These finite models with fixed ends may be relevant for SWNTs connected to the electrodes from both ends. In this case the dangling bonds of free-end carbon atoms are combined with electrode states. Since this is a finite system all the parameters of calculation including supercell size are reoptimized as discussed in Section III. Fe atoms remain stable for $\theta=1 / 2$ and 1 geometries, but one $\mathrm{Fe}$ atom is detached from the wire for $\theta$ $=2$ geometry and is attached to $\mathrm{C}$ atom at fixed ends. For a finite but longer system this effect will be minute. The ground state of all the systems are found to be ferromagnetic with total magnetic moments, $\mu_{T}=1.9,4.0$, and $5.4 \mu_{B}$ for $\theta=1 / 2,1$, and 2, respectively. Magnetic moment per Fe decreased with increased $\mathrm{Fe}-\mathrm{Fe}$ interaction at $\theta=2$. The results indicate that ferromagnetic ground state will be conserved for finite systems. When the number of states near $E_{F}$ is compared with TDOS of infinite counterparts (see Fig. 6) we also notice some changes in the distribution of spin states. These changes occur since first, the electronic structure of bare nanotube changes due to fixed open ends. Second, the interaction between $\mathrm{Fe}$ and $\mathrm{C}$ atoms at both ends affects the electronic structure. Nevertheless, as the length of a finitesize system increases, the discrete electronic states are expected to converge to the spin-dependent TDOS of infinite and periodic system. On the other hand, the fact that the contribution of minority spin states is relatively larger than that of majority spin states near $E_{F}$ for $\theta=1 / 2$ and 2 is similar to their infinite counterparts yielding high $P\left(E_{F}\right)$.

In the right panels of Fig. 6, we present more realistic systems for finite-size devices. Here we consider slightly longer segments of the $(8,0)$ SWNT and let the carbon atoms at both ends relax to close. These segments are comprised of 96 carbon atoms for $\theta=1 / 2$ and $\theta=1$, but 160 carbon atoms for $\theta=2$. By adsorbing $\mathrm{Fe}$ atoms similar to the cases of $\theta$ $=1 / 2,1$, and 2 , we examined geometry and then calculated spin-polarized electronic structure and magnetic moments. While Fe atoms remain stable for $\theta=1 / 2$ and 1 , the Fe chain which is composed of 8 atoms for $\theta=2$ has deformed due to end effects and strong $\mathrm{Fe}-\mathrm{Fe}$ interaction. For longer SWNTs this effect is expected to be minute and in any case the ferromagnetic ground state is conserved with $\mu_{T}=10 \mu_{B}$ like the stable low doping cases $\theta=1 / 2$ and 1 . The energy level diagram of spin states and total magnetic moments of those $\mathrm{Fe}$ adsorbed needles are strongly dependent on the number of $\mathrm{Fe}$ atoms, and on their adsorption geometry. Moreover, we see dramatic changes between left panels (corresponding to fixed ends) and right panels (corresponding to closed ends). The spin polarization and the ferromagnetic ground states are expected to be maintained even after these finite systems are connected to the nonmagnetic metal electrodes from both sides. Depending on the character of the contact and type of the metal, the discrete levels can shift and can form resonances. Under an applied electric field these ferromagnetic needles behave as a resonant tunneling device, as well as a spin valve for different spin directions. The size of the SWNT segment and the geometry of decoration of TM atoms, as well as their type can be relevant parameters to engineer nanospintronic devices.

\section{CONCLUSION}

This paper presented a systematic analysis for the stability, atomic, electronic, and magnetic properties of TM atomic chains adsorbed on the external and internal wall of the $(8,0)$ SWNT. For the sake of comparison we also considered bare TM chains by removing SWNT. The effects of coverage, 


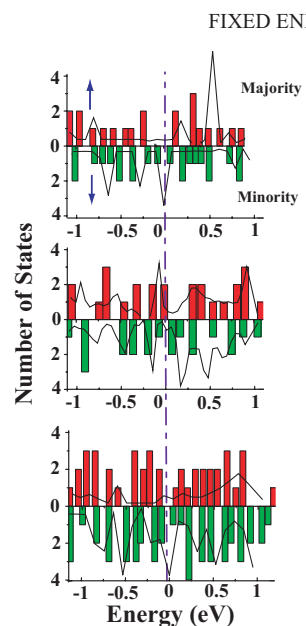

(a)

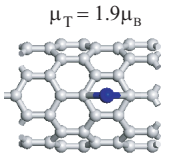

(b) $\mu_{\mathrm{T}}=4.0 \mu_{\mathrm{B}}$

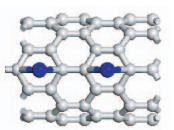

(c) $\quad \mu_{\mathrm{T}}=5.4 \mu_{\mathrm{B}}$

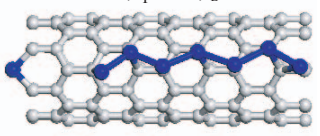

CLOSED ENDS

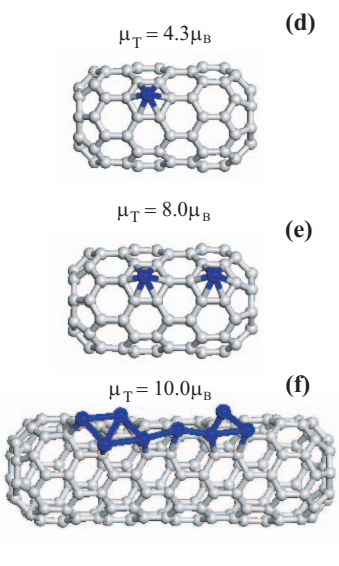

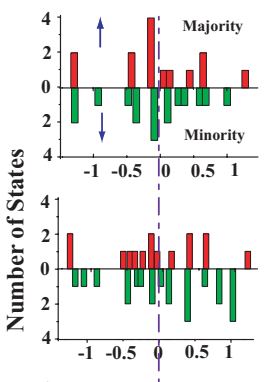

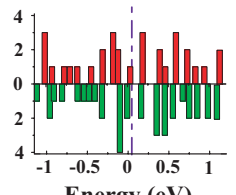

FIG. 6. (Color online) Left panels: The number of states and corresponding structures of Fe atoms adsorbed on a finite $(8,0)$ SWNT with open but fixed ends for (a) $\theta=1 / 2$ (i.e, 64 carbon atoms + one $\mathrm{Fe}$ ), (b) $\theta=1$ (i.e, 64 carbon atoms $+2 \mathrm{Fe}$ ), and (c) $\theta=2$ (i.e, 128 carbon atoms $+8 \mathrm{Fe}$ ). The zero of energy is set to the Fermi level $E_{F}$. The total net magnetic moment for each finite tube, $\mu$, is shown. Total density of states of majority and minority spin states of infinite and periodic systems are also shown by continuous lines for $\theta=1 / 2,1$, and 2 . Right panels: Atomic configurations of Fe atoms adsorbed on the finite size $(8,0)$ SWNT with closed ends. (d) One Fe atom is adsorbed on a tube consisting of 96 carbon atoms. (e) Two Fe atom are adsorbed on a tube consisting of 96 carbon atoms. (f) Eight Fe atoms are adsorbed on a tube consisting of 160 carbon atoms. The calculated total magnetic moments $\mu_{T}$ and TDOS of majority and minority spin states are illustrated for each configuration.

geometry of the adsorbed chain configuration, and the size of the tube on the magnetic and electronic properties have been investigated. We found that all adsorbed chains have ferromagnetic ground state. The coupling among the adsorbed TM atoms and the charge transfer between adsorbed TM and nearest carbon atom of SWNT play an important role in determining the resulting magnetic moment. Usually, the magnetic moment of the free TM atom is reduced upon the adsorption. We found that high spin polarization at the Fermi level can be obtained by the adsorption of $\mathrm{V}$ and Fe chains on the $(8,0)$ SWNT at specific geometries. The polarization values achieved as high as $90 \%$ are expected to be suitable for nanospintronic application. Interesting variation of the magnetic moment and binding energy with the number of filled $d$ electrons of the adsorbate have been revealed. The dependence of the magnetic properties, in particular spin polarization, on the radius and band gap of the zigzag tubes, are further investigated by considering TM-chain adsorbed $(6,0)$ and $(9,0)$ SWNTs.

The spin-dependent electronic structure and the net magnetic moment calculated for finite-size systems are found to be different from infinite and periodic systems. Our results suggest that these finite-size tubes holding TM atoms can be used as a nanomagnet and can perform as spin-valve or spindependent resonant-tunneling devices when they are connected to the metal electrodes from both ends. It is demonstrated that the semiconducting carbon nanotubes constitute a suitable substrate to hold transition metal chains and metallic leads to form nanoscale spintronic devices.

\section{ACKNOWLEDGMENTS}

S.C. acknowledges the financial support of TÜBA. We thank Dr. Sefa Dag for valuable discussions.
*Electronic address: ciraci@fen.bilkent.edu.tr

${ }^{1}$ K. Tsukagoshi, B. W. Alphenaar, and H. Ago, Nature (London) 401, 572 (1999).

${ }^{2}$ S. Ciraci, S. Dag, T. Yildirim, O. Gülseren, and R. T. Senger, J. Phys.: Condens. Matter 16, R901 (2004).

${ }^{3}$ S. B. Fagan, R. Mota, A. J. R. da Silva, and A. Fazzio, Phys. Rev. B 67, 205414 (2003).

${ }^{4}$ S. B. Fagan, R. Mota, A. J. R. da Silva, and A. Fazzio, Microelectron. J. 34, 481 (2003).

${ }^{5}$ S. B. Fagan, R. Mota, A. J. R. da Silva, and A. Fazzio, J. Phys.: Condens. Matter 16, 3647 (2004).

${ }^{6}$ C. K. Yang, J. Zhao, and J. P. Lu, Phys. Rev. Lett. 90, 257203
(2003).

${ }^{7}$ M. S. Ferreira and S. Sanvito, Phys. Rev. B 69, 035407 (2004).

${ }^{8}$ E. Durgun, S. Dag, V. M. K. Bagci, O. Gülseren, T. Yildirim, and S. Ciraci, Phys. Rev. B 67, 201401(R) (2003); E. Durgun, S. Dag, S. Ciraci, and O. Gülseren, J. Phys. Chem. B 108, 575 (2004).

${ }^{9}$ A. T. Costa, D. F. Kirwan, and M. S. Ferreira, Phys. Rev. B 72, 085402 (2005).

${ }^{10}$ B. Zhao, I. Monch, T. Muhl, and C. M. Schneider, Appl. Phys. Lett. 80, 3144 (2002).

${ }^{11}$ S. J. Tans, M. H. Devoret, H. Dai, A. Thess, R. E. Smalley, L. J. Geerlings, and C. Dekker, Nature (London) 386, 474 (1997). 
${ }^{12}$ S. Frank, P. Poncharal, Z. L. Wang, and W. A. de Heer, Science 280, 1744 (1998).

${ }^{13}$ C. Yang, J. Zhao, and J. P. Lu, Nano Lett. 4, 561 (2004).

${ }^{14}$ Y. Yagi, T. M. Briere, M. H. F. Sluiter, V. Kumar, A. A. Farajian, and Y. Kawazoe, Phys. Rev. B 69, 075414 (2004).

${ }^{15}$ Half-metallic properties have been found also in linear chains of carbon atoms which are periodically doped, either by $\mathrm{Cr}$ or $\mathrm{Co}$ atom. See, S. Dag, S. Tongay, T. Yildirim, E. Durgun, R. T. Senger, C. Y. Fong, and S. Ciraci, Phys. Rev. B 72, 155444 (2005).

${ }^{16}$ M. C. Payne, M. P. Teter, D. C. Allen, T. A. Arias, and J. D. Joannopoulos, Rev. Mod. Phys. 64, 1045 (1992).

${ }^{17}$ Numerical computations have been carried out by using VASP software: G. Kresse and J. Hafner, Phys. Rev. B 47, 558 (1993); G. Kresse and J. Furthmuller, Phys. Rev. B 54, 11169 (1996).

${ }^{18}$ W. Kohn and L. J. Sham, Phys. Rev. 140, A1133 (1965); P. Hohenberg and W. Kohn, Phys. Rev. 136, B864 (1964).

${ }^{19}$ D. Vanderbilt, Phys. Rev. B 41, R7892 (1990).

${ }^{20}$ J. P. Perdew, J. A. Chevary, S. H. Vosko, K. A. Jackson, M. R. Pederson, D. J. Singh, and C. Fiolhais, Phys. Rev. B 46, 6671 (1992).

${ }^{21}$ J. P. Perdew, K. Burke, and M. Ernzerhof, Phys. Rev. Lett. 77, 3865 (1996).

${ }^{22}$ M. Methfessel and A. T. Paxton, Phys. Rev. B 40, 3616 (1989).

${ }^{23}$ In order to ensure a spacing that hinders significant interaction between nearest SWNTs we took $a_{s c}=b_{s c}=14,16$, and $17 \AA$ for $(6,0),(8,0)$, and $(9,0)$ SWNT, respectively.

${ }^{24}$ The optimum values of cutoff energy of plane waves and number of $\mathbf{k}$ points used in the present calculations are tested for experimentally well-known crystals such as diamond for carbon as well as for relevant carbon nanotubes.
${ }^{25}$ H. J. Monkhorst and J. D. Pack, Phys. Rev. B 13, 5188 (1976).

${ }^{26}$ For the definition of the radial strain $\epsilon_{r}$, see, for example, O. Gülseren, T. Yildirim, S. Ciraci, and C. Kilic, Phys. Rev. B 65, 155410 (2002).

${ }^{27}$ All magnetic moments $\mu$ discussed in the paper are given per TM atom unless otherwise mentioned.

${ }^{28}$ Y. Zhang and H. Dai, Appl. Phys. Lett. 77, 3015 (2000); Y. Zhang, N. W. Franklin, R. J. Chen, and H. Dai, Chem. Phys. Lett. 331, 35 (2000).

${ }^{29}$ A. N. Andriotis, M. Menon, and G. E. Froudakis, Phys. Rev. B 62, 9867 (2000).

${ }^{30}$ X. Blase, L. X. Benedict, E. L. Shirley, and S. G. Louie, Phys. Rev. Lett. 72, 1878 (1994).

${ }^{31}$ It is very well known that a SWNT with small radius, that can be viewed as the graphene sheet rolled over a cylinder of radius $R$, behaves differently from the planar graphene (see Ref. 30). On the other hand, adsorption properties of a SWNT with very large radius can be taken as those of planar graphene corresponding to $R \rightarrow \infty$.

${ }^{32}$ D. M. Duffy and J. A. Blackman, Phys. Rev. B 58, 7443 (1998).

${ }^{33}$ The geometry and $d_{\mathrm{C}-\mathrm{Fe}}$ calculated by PW91 are practically the same as PBE, but $\mu$ values slightly change and become 2.4, 4.0, and $3.0 \mu_{B}$ for $\theta=1 / 2,1$, and 2, respectively, for PBE.

${ }^{34} \mathrm{~J}$. Friedel, The Physics of Metals, edited by J. M. Ziman (Cambridge University Press, New York, 1969); D. G. Pettifor, in Solid State Physics, edited by H. Ehrenreich and D. Turnbull (Academic, New York, 1987), vol. 40, p. 43.

${ }^{35}$ O. Gülseren, T. Yildirim, and S. Ciraci, Phys. Rev. B 65, 153405 (2002). 